characteristics. RESULTS/ANTICIPATED RESULTS: Mean age of sample was 56.5 years, $57.3 \%$ female, $54.9 \%$ white, $18.9 \%$ black and $13.1 \%$ Hispanic; $64.3 \%$ were discharged home, $15.8 \%$ to a skilled nursing or other intermediate care facility, $15.5 \%$ to home with home care and $2.4 \%$ left against medical advice. The top 3 discharge diagnoses were vaginal delivery (6.3\% of discharges), psychosis $(4.7 \%)$, and major joint replacement $(2.9 \%)$. In adjusted analysis compared to white patients, black and Hispanic patients did not have an risk of increased LOS after being discharged to non-home destinations vs. discharged home, (black patients, adjusted OR [AOR], 0.97; 95\% CI: 0.94-1.00, $\mathrm{p}=0.08$; Hispanic patients, AOR, 1.01; 95\% CI: $0.98-$ $1.05, \mathrm{p}=0.5)$. However, being black compared to white and discharge to non-home destinations significantly increased LOS. DISCUSSION/SIGNIFICANCE OF IMPACT: In this large sample of patients admitted for inpatient care in 2014 in New York, we found no independent effect between race and discharge destination on a patient's LOS after controlling for patient, disease and between-hospital characteristics. However race/ethnicity increased LOS, suggesting its effect may play a role on in-hospital processes CONFLICT OF INTEREST DESCRIPTION: Dr. Ghosh has no relevant relationships with commercial interests to disclose Dr. Ibrahim has no relevant relationships with commercial interests to disclose

4131

\section{Recruitment and Retention of Individuals with a Cocaine} Use Disorder

Kate Brown, MSN, NP ${ }^{1}$, and Bernadette Capili

${ }^{1}$ Rockefeller University

OBJECTIVES/GOALS: 1) illustrate the varied challenges individuals with a cocaine use disorder experience in daily life, 2) demonstrate techniques for empathizing and building rapport with potential subjects, and 3) identify recruitment obstacles and solutions. METHODS/STUDY POPULATION: Methods: We use a multisource strategy to recruit our participants and employ practical techniques to enhance protocol adherence. Methods include a welcoming environment, establishing a routine with flexibility, personalized attention, and incentives for participation. Study population: Individuals with a cocaine use disorder. RESULTS/ ANTICIPATED RESULTS: Understanding the life of an individual with a cocaine use disorder is paramount to successful recruitment and retention in addiction research studies. Our clinicians have been able to recruit and retain participants successfully by employing empathetic interpersonal skills, personalized attention, and healthrelated incentives. DISCUSSION/SIGNIFICANCE OF IMPACT: The Centers for Disease Control and Prevention estimated that 69,029 people died of a drug overdose during the period from February 2018 to February 2019, with 23\%, due to cocaine. While methadone and buprenorphine-naloxone maintenance treatment allow opioid-dependent individuals achieve a sense of physical and mental stability, there is no pharmaceutical treatment to help a cocaine-dependent individual cope with cravings or the depression and anxiety that typically follow a cocaine binge. The development of a cocaine use disorder is multi-factorial and presents a significant challenge in terms of discovering treatments, identifying efficient recruitment and retention strategies is the first step for effective research.
Researchers' Experiences Working With Community Advisory Boards: How Community Member and Patient Stakeholder Feedback Impacted The Research

Tabetha A. Brockman ${ }^{1}$, Monica L. Albertie ${ }^{1}$, Noreen A. Stephenson ${ }^{1}$, Sumedha G. Penheiter, $\mathrm{PhD}^{1}$, and Christi A. Patten, $\mathrm{PhD}^{1}$ ${ }^{1}$ Mayo Clinic

OBJECTIVES/GOALS: To assess researchers' experiences working with community advisory boards (CABs) and how community member/patient stakeholder feedback impacted the research. METHODS/STUDY POPULATION: Researchers $(\mathrm{N}=34)$ who presented their research to a Mayo Clinic CAB (at MN, AZ, or FL) from 2014-2017 were invited to participate in an interview inperson or by phone averaging 10-15 min. Researchers were asked "In what ways did the feedback you received from the CAB influence your research?" The validated structured 7 -item interview included domains assessing potential influence that CABs had on the research: 1) Pre-research (e.g., generated ideas), 2) Infrastructure (e.g., budget preparation), 3) Research design, 4) Implementation (e.g., research recruitment), 5) Analysis, 6) Dissemination, and 7) Post-research (e.g., assist in formulating next steps). RESULTS/ANTICIPATED RESULTS: 17 interviews were completed (8 no longer at Mayo, 9 no response). Researchers presented their study to a $\mathrm{CAB}$ a mean of 4 years (range 3-5) before the interview. Researchers reported that the $\mathrm{CAB}$ had influenced their research in the following domains: $24 \%$ in pre-research, $24 \%$ infrastructure, $41 \%$ study design, $41 \%$ implementation, $6 \%$ analysis, $24 \%$ dissemination, and $18 \%$ for postresearch activities. The mean total score was $=1.8(\mathrm{SD}=1.7$, range $0-6$ ), of a possible range of 0-7. DISCUSSION/SIGNIFICANCE OF IMPACT: Impact of CAB feedback on the research was moderate. Ways to enhance impact could include follow-up with researchers and $\mathrm{CAB}$ members.

4295

\section{The Impact of Social Determinants of Health on Hepatocellular Carcinoma Outcomes \\ Lauren Devore Nephew ${ }^{1}$, Susan Rawl, Archita Desai, Eric Orman, Marwan Ghabril, Kavish Patidar and Naga Chalasani \\ ${ }^{1}$ Indiana Univeristy School of Medicine}

OBJECTIVES/GOALS: Achieving therapy for hepatocellular carcinoma (HCC) involves navigating through a complex cascade of care. Non-HCC cancer mortality has been associated with social determinants of health outside of cancer specific risk. Our objective is to explore the impact of social determinants on HCC outcomes. METHODS/STUDY POPULATION: Patients with HCC were enrolled from 3 hospitals form June, 12019 to December 1, 2019. A chart review was done to collect information on liver disease severity and cancer stage. Patients were interviewed to collect information on the following: 1) socioeconomic status (income, education, insurance status, and employment status), 2) literacy (Rapid Estimate of Adult Literacy in Medicine (REALM-R) and Brief Health Literacy Screening Tool (BREIF)), 3) social support (Patent Reported Outcome Measurement Information System (PROMIS) instrumental and information support tool), 4) quality of life (PROMIS global and mental health tool), 5) substance abuse, and 6) linkage to care. RESULTS/ANTICIPATED RESULTS: Data 
compiled on the social determinants of health revealed $(\mathrm{n}=35): 1$ ) $60.0 \%$ of patients had incomes below $\$ 30,000$ per year, $60.0 \%$ of patients had not gone past high school for education, and $8.6 \%$ had full time employment, 2) the average BREIF score was 10.3 (range 3-15)(4-12 indicate limited literacy). The average REALM$\mathrm{R}$ score was 5.5 (range $0-8)$ ( $<6$ indicate at risk for poor literacy), 3) patients had strong instrumental (T score 61.4 \pm 7.1 ) and information social support ( $\mathrm{T}$ score $64.6 \pm 4.7$ ) (mean $\mathrm{T}$ scores calibrated to a general population mean of 50), 4) patients had poor mental (T score $43.7 \pm 6.5$ ) and physical quality of life (T score $46.6 \pm 9.9$ ), 5) $25.7 \%$ of patients reported alcohol use in the past 90 days 6) $80.0 \%$ of patients reported that their doctor had spoken to them about liver transplantation. DISCUSSION/SIGNIFICANCE OF IMPACT: This patient population was well linked to care with good social support. However their literacy, socioeconomic status, mental and global health was poor and substance use history complex. Continued follow up of this cohort is planned to determine how these factors might impact their ability to navigate through the care cascade as well as survival.

The Prevalence of Food Insecurity Among University of Utah Medical Students: Documenting the Need for Supportive Programs

Alyssa Thorman ${ }^{1}$, and Harneet Kaur Dhillon ${ }^{2}$

${ }^{1}$ University of Utah School of Medicine; ${ }^{2}$ University of Utah Medical School

OBJECTIVES/GOALS: Undergraduates experience food insecurity at rates $21 \%$ higher than the general population. Because professional students have been omitted from these studies, the goal of this project is to determine the prevalence of food insecurity among medical students at one academic institution. METHODS/ STUDY POPULATION: A cross-sectional research design was used to quantify the food insecurity status of medical students at the University of Utah. The USDA's validated 6-item Food Security Survey Module was distributed via email to all currently matriculated medical students. Student's responses were anonymous but questions about gender and age were included. Respondents $(\mathrm{N}=200)$ were scored per the module as food secure, food insecure, or very low food security. RESULTS/ANTICIPATED RESULTS: Statistical analysis included frequencies and chi-square tests. Medical students $(\mathrm{N}=166)$ showed $50.6 \%$ of respondents experienced food insecurity in the past 12 months, $16.3 \%$ experienced very low food security. While there were no significant relationships between food security status and gender or age, general trends did show divorced and separated students had higher food insecurity risk 82\%. A similar study in 2014 surveyed undergraduates at the same location; $51 \%$ of respondents $(\mathrm{N}=221)$ experienced food insecurity. While medical students experience food insecurity at rates much higher than the national average, prevalence is lower than undergraduates at the same institution. DISCUSSION/ SIGNIFICANCE OF IMPACT: Burnout and suicide in medical training are at an all-time high; professional and academic pursuits are limited when physiological needs of food security are not being met. Study results suggest, $50 \%$ of respondents are food insecure. This should inform the development of supportive programs.
4240

The relationship between family structure and quality of life among children with asthma

Magda Shaheen ${ }^{1}$, Arneshia LA'Shelle Bryant-Horn², and Senait Teklehaimanot $^{2}$

${ }^{1}$ David Geffen School of Medicine at UCLA; ${ }^{2}$ Charles R Drew University

OBJECTIVES/GOALS: Asthma is a life-long, chronic lung disease that inflames and narrows the airways. Its effects on quality of life in children can be exacerbated. The goal of this study was to investigate the link between asthma, family structure and demographics and how it impacts quality of life in children. METHODS/STUDY POPULATION: We analyzed data from a cross sectional study of the 2016-2017 National Survey of Children's Health, NSCH, to assess the relationship between determinants of health variables and the outcome variable of parent's report of child's perceived health status (quality of life). The study population was children under the age of 18. Data were analyzed using descriptive, bivariate analysis using Chi square, and multiple logistic regression of quality of life and family structure adjusting for confounding variables. RESULTS/ ANTICIPATED RESULTS: The study included 5,687 children. Significant predictors of asthmatic children's quality of life were severity of asthma, self-perceived mental/physical health status of adults, neighborhood safety for children $(\mathrm{p}<0.05)$. The interaction between family structure and asthma severity was significant indicating that asthma severity was an effect modifier. Among children with mild asthma, predictors of quality of life were self-perceived mental/ physical health status of adults in the household, neighborhood safety of children, physical activity status of children $(\mathrm{p}<0.05)$. Among children with severe asthma, predictors were family structure and physical/mental health of adults $(\mathrm{p}<0.05)$. DISCUSSION/ SIGNIFICANCE OF IMPACT: This study suggests children with severe asthma who are born to single mothers with lower parental reporting of physical/mental health status had a lower quality of life. A longitudinal study could be implemented to target these three measures to improve quality of life among these children. Also, a culturally adapted intervention involving community, parents, and providers is needed to improve the quality of life of the children with asthma.

4408

Using a human-centered design process to address challenges of engaging pregnant \& parenting women with opioid use disorder

Sarah Wiehe ${ }^{1}$, Dustin Lynch², Courtney Moore², Brandon Cockrum², Bridget Hawryluk ${ }^{2}$, and Gina Claxton ${ }^{2}$

${ }^{1}$ Indiana University School of Medicine; ${ }^{2}$ Indiana Clinical and

Translational Sciences Institute

OBJECTIVES/GOALS: Using a human-centered approach, IDEO, a nationally-renown human-centered design team, and Research Jam, Indiana CTSI's patient engagement core, integrated and tailored complimentary programs to address the challenges of engaging mothers with opioid misuse around the time of birth. METHODS/STUDY POPULATION: Gathered data through focus groups, site visits, and one-on-one interviews with key stakeholders: mothers in opioid use recovery, peer recovery coaches, and other 